\title{
Living Labs: A Bibliometric Analysis
}

\author{
Dr. Shane McLoughlin \\ LERO, NUIM \\ shane.mcloughlin@mu.ie \\ Dr. Giovanni Maccani \\ LERO, NUIM \\ giovanni.maccani@mu.ie
}

\author{
Dr. David Prendergast \\ Intel Corp \\ david.k.prendergast@intel.com
}

\author{
Prof. Brian Donnellan \\ LERO, NUIM \\ brian.donnellan@mu.ie
}

\begin{abstract}
The objective of this study is to understand how Living $L a b(s)$ (LL) as a concept and research approach has developed, proliferated and influenced scholarly research to date. The goal is in assisting both the $L L$ and Action Design Research (ADR) communities in advancing both fields by establishing understanding, commonalities and challenges in advancing both research agendas. We adopt a bibliometric methodology to understand the scholarly impact, contribution and intellectual structure of $L L$ as a new approach to innovation. We conclude with recommendations on advancing both $A D R$ and $L L$ fields of research, highlighting that increased crosscollaboration going forward offers clear opportunities to both fields.
\end{abstract}

\section{Introduction}

Within Information Systems (IS) research, Living Lab (LL) methodologies have been discussed as an opportunity to extend the focus of Design Science Research (DSR)[14]. In this respect, the LL approach can be viewed as an 'exemplar' [24] of "the growing interest in conceptualizing the artifact in sociotechnical terms...interwoven with organizational and social elements"[10]. Advancing work by Thapa et al.[24] and Coenen et al. [7], Maccani et al. [15] compared LL and ADR methodologies, concluding much congruency as well as challenges in aligning the goals of ADR and LL research. They highlighted the opportunities for LL by integrating ADR methodology, which would serve to benefit both fields of research. This current study advances such efforts by mapping the intellectual evolution of LL research, and is the first such study contributing a bibliometric analysis of LL. Living Labs as a process, methodology and/or environment for innovation [1], began to proliferate in the academic literature over the past 10 years. Whilst its origin as a concept can be principally traced to MIT technology research benefiting from re-creating real life home environments, it later became more widely used within academic literature via research arising out of the EnoLL (European Network of Living Labs) research community. With EnoLL, the focus moved beyond the home setting to developing innovations in other real life environments, with associated definitions, theoretical lenses and methods applied.

Whilst LL research has clearly gained some traction (e.g. [12]) it appears LL has not yet entered the mainstream Information Systems (IS) literature. In this way, recent scholars suggest that "a theoretical and methodological gap continues to exist in terms of the restricted amount and visibility of Living Lab literature and its contributions to research [2]. Therefore, the objective of this study is to understand how Living Labs (LLs) as a concept and research approach has developed, proliferated and influenced scholarly research. Such an understanding is important in establishing the value of LL, where LL research needs to go from here, and how researchers interested in a LL approach can best contribute. For example, this study will aid both LL and ADR scholars in identifying promising or diminishing fields of research, important avenues for publication, as well as the core authors and work influencing the LL field.

To address the objective of this study, we propose a bibliometric analysis of Living Labs literature. Originating in the discipline of Library and Information Science, bibliometric research concerns the use of descriptive and statistical analysis of academic publications meta-data in order to understand the evolution and/or impact of knowledge domains.

In the following sections, we begin by overviewing LLs as a concept and research approach, as well as its cited rationale and benefits. Next, we outline our methodology to address our research questions. Our findings are structured according to the three key research questions below:

1. What is the scholarly impact of Living Lab as a concept and/or approach?

a. How much traction has LL as a concept and/or approach gained in the academic community?

b. What are the publication venues and disciplines associated with Living Lab influenced research? 
c. What is the geographic distribution of LL influenced research?

2. What are the research trends associated with Living Lab influenced research, and how have they evolved over time?

3. How has the intellectual structure of LL influenced research matured?

We conclude with discussion and recommendations based on our findings.

\section{Living Labs}

'Living Lab' has emerged in the past ten years as a new approach for innovation. A recent definition provided by EnoLL (European network of Living Labs) refers to "user-centered, open innovation ecosystems based on a systematic user co-creation approach, integrating research and innovation processes in real life communities and settings" [18]. The basic premise of LLs is to study and involve end users in the innovation process in more naturalistic settings through exploration and/or evaluation [16] of artifacts. This may involve rich life contexts, as well as alternative and competing pre-existing solutions in place. Users play multifaceted roles and involvements, from offering ideas to validating designs. Furthermore, LLs often involve a quadruple helix approach, with all stakeholders participating in developing solutions. The demonstration, development and validation of technology is carried out in real world environments to shape technology development, thereby increasing expected value and adoption upon market release.

The emergence of LL as a methodology for developing ICT has been a response to shortcomings in the more standardized 'test-bed' approaches for successful products, whereby benefits should be shown as a result of proposed technological solutions [16]. In some cases, LLs are the next stage in the product development lifecycle, after test-bed research, and are amongst a variety of distinct approaches for developing ICT solutions [18]. Furthermore, to support LL approaches, there exists a taxonomy of $\mathrm{LL}$ configurations from corporate LL set-ups to academic or intermediary LL. Here, LLs move beyond a methodology to panel infrastructures and organizations set-up to accommodate LL experimentation (e.g. Laurea (Finland), Suslab (cross-European).

In terms of core benefits, LLs are an opportunity to understand the benefit or 'value' of ICT before business mechanisms such as 'dependency' and 'lock in' [18] upon going to market. By experimenting in real life environments, rich, complex and often unforeseen circumstances are factored in to developing solutions. They provide an opportunity to carry out comparative use-cases across differing socio-cultural sites, in order to generalize findings, and ensure wider adoption. Involving end users in product development furthermore serves to reduce risk of R\&D investment. From a city governance and service provision perspective, LL approaches enable cities to ensure some control, planning and levels of commitment to business developments and initiatives, as well as ensure citizens are involved in the development of solutions that affect their lives.

\section{Method}

The basis for our bibliometric analysis began with carrying out a Systematic Literature Review on Living Lab titled papers in order to (1) ascertain key LL terms for searching LL (influenced) papers, and (2) identify highly cited LL conceptual/methodological papers for further bibliometric analysis. The Systematic Literature Process adhered to Okoli's 8 step methodology [19]. The 8 steps are: (1) Purpose of the Literature Review, (2) Protocol and Training, (3) Searching for the Literature, (4) Practical Screen, (5) Quality Appraisal, (6) Data Extraction, (7) Synthesis of Studies, and (8) Writing the Review. The Review Question to guide this process was stated as, "What are the conceptual/methodological approaches to LL research?'

According to Okoli [19], both specific and general subject databases should be considered for searching the literature. Data was collected from 3 key databases for searching LL work in February 2017, the 'AIS basket of eight', 'Scopus' and Google Scholar (GS). We used Harzing's Publish or Perish (PoP) to retrieve GS data. In total, we retrieved 1600 GS and 382 Scopus articles using the search criteria of 'Living $\mathrm{Lab}(\mathrm{s})$ ' in the title. The search across the "AIS Basket of 8" did not produce any result. We restricted our search to 'title' to capture only strongly based LL papers, as GS does not facilitate keyword and abstract searching. After removing duplicates and non-English articles, we arrived at 1,143 (1043 unique GS and 40 unique Scopus articles) papers from Scopus and GS. Screened papers by analyzing titles based on the RQ left 427 articles to further consider. We subsequently read abstracts and arrived at 169 papers that we deemed had a strong conceptual/methodological focus. Consistent with the lack of LL-related publications in the mainstream literature, we did not undertake further quality appraisal of the papers as a further exclusion criterion, beyond selecting peer reviewed conference and journal papers only. Thus, 169 papers (Dataset A) were systematically ordered and selected for this study.

In order to address all the research questions, bibliometric data from different databases offered 
strengths and weaknesses depending on the particular research question posed. 'Dataset A' formed the basis for carrying out additional steps to produce two bibliometric datasets for analysis; 'Dataset B' and 'Dataset C'. 'Dataset B' concerns a wider bibliometric dataset obtained from Google Scholar (casting the net wide). 'Dataset C' concerns a more in-depth dataset obtained from Scopus (casting the net deep) because of the complete bibliometric data it provides. We chose Scopus over 'Web of Science' (WoS) because of its wider coverage, particularly in relation to Information and Computer Science related topics [25].

To identify 'Dataset B', we first extracted all LL definitions from 'Dataset $A$ ' to identify key terms associated with LL research. 111 papers (from 'Dataset A') offered one or more definitions of LL. We analyzed all LL definitions found in these papers, and selected the terms, 'Innovation' $(\mathrm{n}=120)$, 'Real life' $(\mathrm{n}=103)$ and 'User(s)' $(\mathrm{n}=96)$, which could be found in almost all definitions retrieved. Limiting our search to these terms would help screen out irrelevant results (as initial screening of 'Living Lab(s) searching found non-related articles in the biological and other sciences). We chose not to include the terms 'environment', 'Co-Creation', 'methodology', 'partnership' and 'stakeholder', as they were referred to by half or less LL definitions. We then searched 'Google Scholar' using the terms, 'Living Lab(s)' AND 'innovation' AND 'real life' AND user(s), anywhere in the article GS can search. After removing duplicates, we ascertained 2533 articles (Dataset B).

To identify 'Dataset C', we first drew on 'Dataset A' to examine the citation patterns from GS. Figure 1. breaks down papers by citation count, and shows that 134 of the papers received citations with a combined total of 3291 citations, though a small number of papers received most citations. This shows that the citation patterns of LL papers conform to distribution patterns observed in other bibliometric studies [21].

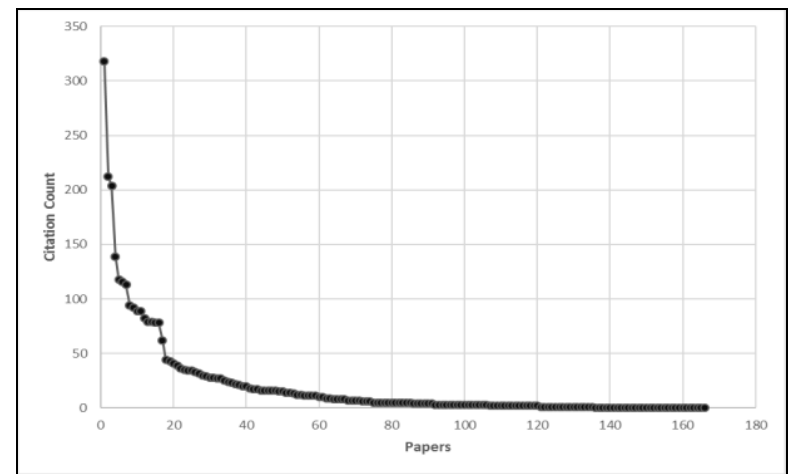

Figure 1. Citation counts of LL conceptual/Methodological papers
Upon examination of citation counts (shown in Figure 1.0), we extracted 60 top cited papers. To ensure older papers were not unduly biased in our selection, we included 20 of our 60 top cited papers based on the top 5 cited papers for each of the past four years (2012-2016). Next, we used Scopus to collate all papers who cited any of the top $60 \mathrm{LL}$ conceptual/methodological papers. Scopus found 496 unique papers citing these LL papers (Dataset $\boldsymbol{C}$ ).

To address the research questions, analysis was carried by using a combination of Taporware (for word and multi-word analysis) and Excel for bibliographic analysis and visualization of Dataset B. We used a combination of Excel and Citespace (v. 5.0 R2) for 'Dataset C'. Citespace is a bibliometric analysis and visualization software package developed over the past 10 years by Chen [5] in Drexel, USA. Its strength lies in Term and Co-Citation mapping \& visualization to understand the intellectual evolution of a subject, field or discipline over time. It has been developed based on bibliometric techniques emanating from the Information Science discipline. We used Citespace to understand the scientific structure and knowledge domains of work citing LL key conceptual/methodological papers (i.e. Dataset C). Citespace works by generating a network synthesized from a series of individual networks (Time slices). Each individual network represents articles published in a 1-year time interval (Slice). Thus, Citespace synthesizes these networks to identify clusters of similar research based on similar use of terms or references, and how they evolve over time. Each term or reference is represented by a node, whereby the connectivity between the nodes shows how frequently they are included by the same papers. The size of the node corresponds with how often they accrue. Crucially, the theory is that if two terms or references co-occur together in papers, then it is likely they are associated in some way, and thus inform the position of nodes to clusters. Once mapped, terms or references which tend to co-occur in papers will tend to lie close together in the visualization shown. For a detailed understanding of Citespace tools and methodology, refer to Chen [5].

\section{Findings}

\subsection{What is the Scholarly Impact of $L L$ as a concept and/or approach?}

4.1.1 How much traction has $\mathrm{LL}$ as a concept and/or approach gained in the academic community? 
To address this question, we analyzed 'Dataset B' (i.e. 2533), and present findings in Figure 2. An increased influence of LL amongst the wider scholarly community since 2005 can be observed, peaking in 2014, and showing decline in 2015/2016. Upon investigation of the most significant publication venues identified in Table 1, we found proceedings of 2016 papers had not yet been circulated in some cases, thus likely accounting for this decline. To contextualize these findings, we compared these results with a GS search for 'Action Design Research' (ADR) anywhere in the article (between the period 2005-2016) and retrieved 846 articles (after removing duplicates etc) (See figure 2.). This suggests LL has performed well compared to related approaches to artifact design. It should be noted that LL was already gaining interest within academic literature between 2005 and 2010, whereas ADR has generated more recent attention, and is more focused as a methodology.

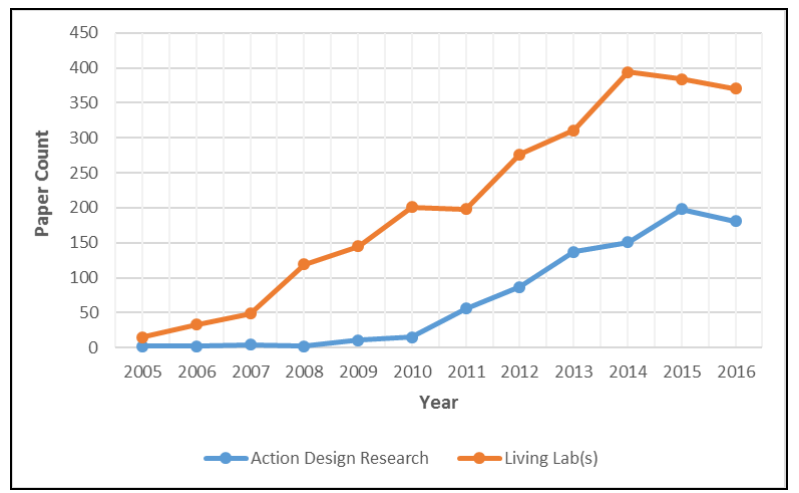

Figure 2. ADR versus LL publications

4.1.2 What are the publication venues and disciplines associated with Living Lab research?

Upon analysis of Dataset C, we found that 39\% of these papers (193 papers) had 'Living Lab(s)' in the title, with a further $5 \%$ (24 papers) including 'Living $\mathrm{Lab}(\mathrm{s})$ ' in keywords, and a further $10 \%$ (50 papers) having 'Living Lab*' in the abstract. Thus, just over half of the papers citing LL conceptual/methodological papers indicated a strong LL focus/component. Of these, 49 papers were found to entail conceptual/methodological papers identified for the basis of this study (i.e. Dataset A).

$37 \%$ of papers $(\mathrm{n}=183$ papers $)$ concerned journal publications, and $51 \%(\mathrm{n}=249$ papers $)$ concerned conferences, with the remainder consisting of book chapters etc. These figures are in line with similar bibliometric studies on the distribution of Computer Science related publication dissemination [25]. A total of 236 publication venues were identified, indicating a heterogeneous dissemination of LL influenced work.
Table 1. Top Publication Venues of LL

\begin{tabular}{|l|c|}
\hline Source & n \\
\hline $\begin{array}{l}\text { International Conference on Engineering, } \\
\text { Technology and Innovation, ICE/ITMC }\end{array}$ & 50 \\
\hline $\begin{array}{l}\text { Lecture Notes in Computer Science (including } \\
\text { subseries on 'Artificial Intelligence' \& } \\
\text { 'Bioinformatics') }\end{array}$ & 35 \\
\hline $\begin{array}{l}\text { IFIP Advances in Information and } \\
\text { Communication Technology journal }\end{array}$ & 9 \\
\hline $\begin{array}{l}\text { Lecture Notes in Business Information } \\
\text { Processing }\end{array}$ & 9 \\
\hline Info Journal & 9 \\
\hline IST Africa & 8 \\
\hline $\begin{array}{l}\text { Proceedings of the Annual Hawaii } \\
\text { International Conference on System Sciences }\end{array}$ & 8 \\
\hline AMCIS conference & 8 \\
\hline $\begin{array}{l}\text { ACM International Conference Proceeding } \\
\text { Series }\end{array}$ & 6 \\
\hline International Journal of Product Development & 6 \\
\hline
\end{tabular}

Table 1. shows where LL influenced papers are disseminated by most, of benefit to researchers becoming engaged in LL research. It highlights that other than the ICE/ITMC conference and 'Lecture Notes in Computer Science', there appears a lack of LL impact concentrated outside of these publication venues. Other significant avenues for LL publication not listed are the 'Technology Innovation Management Review' \& ' Open Living Lab Days' summit.

In terms of disciplinary areas, we assigned publication sources based on the 'All Science Journal Classification' (ASJC) list, and found papers can be broken down as largely 'Computer Science' or 'Information Science' related topics, followed by Business (e.g. Business Management etc.) and Engineering related subjects. See below Table 2. This relates to LLs as a significant approach to technological innovation in business.

Table 2. Disciplinary areas associated with LL

\begin{tabular}{|l|l|}
\hline Disciplinary Area & Total \\
\hline Computer or Information Science & 162 \\
\hline Engineering & 38 \\
\hline Business & 75 \\
\hline Social Science & 11 \\
\hline Arts and Humanities & 11 \\
\hline Other & 40 \\
\hline Not-known & 159 \\
\hline
\end{tabular}

4.1.3. What is the geographic distribution of $\mathbf{L L}$ research? 
There were 422 European affiliated paper contributions ( $85 \%$ of Dataset C), compared to 111 non-European affiliated paper contributions (22\% of Dataset C), with 36 Europe to non-Europe paper collaborations (7\% of Dataset C). A total of 42

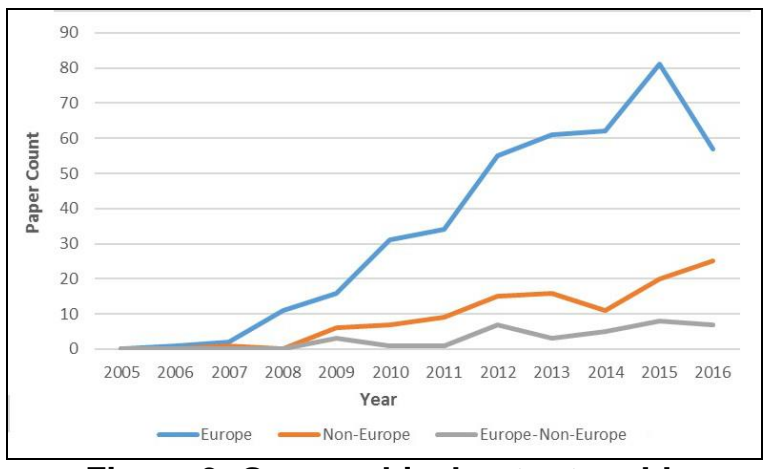

Figure 3. Geographical output on LL influenced research

country affiliated papers were identified (57\% European countries). Within Europe, such efforts are particularly concentrated in Germany (54 papers), Netherlands (51 papers), Finland and France (48 papers each). Whilst the USA (27 papers), Canada (22 papers) and Australia (15 papers) most cite LL conceptual/methodological papers outside of Europe. Thus, LL influenced work is heavily concentrated in Europe, where EU led efforts such as EnoLL have had a clear impact. We found that there has been a steady though modest increase in LL influenced research outside of Europe, compared to Europe where interest has peaked and begun to decline. Figure 3. tracks papers by year, and shows a steady though low number of Europe 'to' non-Europe collaborations. Such findings suggest that 'Living Lab' conceptual/methodological papers are slowly attracting international attention, though more efforts at international collaborations spurred by European expertise in this area can serve to increase its impact.

\subsection{What are the research trends associated with Living Lab influenced research, and how have they evolved over time?}

To address this research question, we adopted two complementary approaches. Firstly, we used 'Dataset B' to cast a wide net over the scholarly landscape, and ascertained research trends using title (multi)word frequency analysis. Secondly, we used Citespace to analyze 'Dataset C' ensuring a deeper more focused analysis of research trends by using combined 'Title' and 'Keyword' terms assigned to papers. Similar approaches have been adopted in other bibliometric studies (See for example; [25] and [5]).

\subsubsection{Term Analysis using 'Dataset B'}

For 'Dataset B', we extracted paper titles and used Taporware to remove 'stop terms' and core LL concepts ('Living Lab(s), Innovation, Real-Life and Co-Creation', partnership and stakeholder) (Based on our analysis of LL definitions)). Figure 4. (displayed at the end of the paper) presents the top twenty terms, and tracks developments across the past six years. It summarizes key trends in research topics and research approaches associated with those concepts. Summaries are derived inductively based on review of titles (and abstracts where necessary) according to terms. It shows that LL influenced work is most commonly associated with such concepts as 'sustainability', 'smart cities', the 'urban' context, and 'open innovation'.

In terms of 'Smart Cities', various concept definitions exist, most referring to renewal and optimization of city governance, services and infrastructure, with many definitions referring to achieving better 'Quality of Life' outcomes for citizens. Much of the literature on Smart Cities tends to emphasize the leveraging of advances in Information and Communication technologies to achieve such 'Smart' outcomes. 'Internet of Things' refers to (multi)sensorial devices deployed in various contexts to either capture and or action data received, in some cases process this that data at the edge, but in all cases be able to transmit and/or receive data via an internet connection. According to Chesbrough [6], 'Open Innovation' is the use of purposive inflows and outflows of knowledge to accelerate internal innovation, and expand the markets for external use of innovation, respectively'. One premise being to draw on external ideas beyond an organization's boundaries in the innovation process.

LL influenced work is most commonly referred to in terms of the 'case study' methodology, whereby trends show, 'co design' is becoming (albeit slowly) more commonly discussed as a method. Co-design, as related to Participatory Design, is an approach to innovation where various stakeholders are involved in the design process. 'Social Innovation' is also slowly gaining traction, and can be defined as a novel solution to a social problem that is more effective, efficient and sustainable than current solutions [4]. Furthermore, the value created primarily accrues to society rather than to private individuals. Such innovations may be products, services or models addressing needs.

A further finding from our analysis is a diminishing focus on the rural context in research, in tandem with an increasingly focus on the urban context. 


\subsubsection{Term Analysis using 'Dataset C'}

Next, we used Citespace for 'Dataset C'. Citespace allowed us to identify term frequency, and clusters of research by mapping networks of cooccurrence in title and keyword terms. This deepens our understanding, providing a more focused and nuanced understanding of knowledge domains, whereby Citespace integrates complete bibliographic information obtained through Scopus.

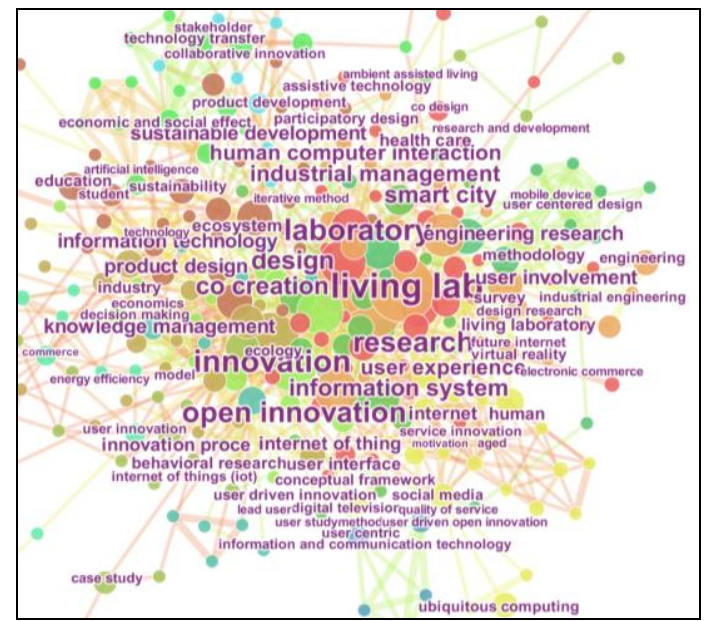

Figure 5. Term Structure Overview

A combined 'Title' and 'Keyword' frequency analysis using Citespace resulted in 21 clusters with a Modularity Q score of .59 and a mean Silhouette score of .51. This suggests the clusters identified are not that well divided into loosely coupled clusters, and that the homogeneity within the clusters is just average. Cluster names assigned in figure 6. are based on ' $\mathrm{Log}$ Likelihood Ratio (LLR). Figure 5. represents the core structure of the network with a visualization threshold of 5 or more occurrences for each term applied. The relative size of terms indicates their frequency relative to other terms. Examining the term structure, we can observe that 'terms' can largely be broken down by 'disciplinary area', specific 'research topic trends' and 'methodological approaches'. Findings highlights how 'Smart city' ( $\mathrm{n}=33)$, 'Sustainable Development' $(\mathrm{n}=$ $18)$ or Sustainability $(\mathrm{n}=7)$, 'Information System' $(\mathrm{n}=$ 30) 'Information Technology' $(\mathrm{n}=16)$, 'Internet of Thing(s)' (IoT) $(\mathrm{n}=20)$ or 'Ambient Intelligence' $(\mathrm{n}=$ $6)$ and 'ubiquitous Computing' $(\mathrm{n}=7)$, 'Health Care' $(\mathrm{n}=14)$ as well as 'Ambient Assistive Living' $(\mathrm{n}=7)$ and 'Assistive Technology' $(\mathrm{n}=7)$ are research topics influenced by LL conceptual/methodological papers.

Within the domain of 'Smart Cities' research, there have been increasing efforts to adopt a LL approach to designing and deploying new urban data/IoT solutions to address city challenges. For example, how IoT sensors can be deployed in a city to monitor and regulate traffic, parking, pollution and water management. Such efforts include FIRE initiatives [8] such as the 'Organicity' project [9], which aims to provide an 'Experimentation As a Service' model for urban data/IoT development. The popularity of LL approaches to 'Smart City' challenges relates to the complex real life context in which IoT solutions must operate, as well as the opportunity to ensure citizen engagement and input in devising solutions that affect them.

In terms of theoretical/methodological design, 'Open Innovation' ( $\mathrm{n}=62)$, 'User Experience' $(\mathrm{n}=20)$, 'User Involvement' $(\mathrm{n}=20)$, 'Participatory Design' $(\mathrm{n}=10)$, 'User Centered Design' ( $\mathrm{n}=8)$, 'Co-Design' $(\mathrm{n}=5)$ most frequently occur in addition to 'Co-Creation' ( $\mathrm{n}=$ 39). Importantly, all of these approaches emphasis the end user in the design process, and in some approaches (such as Co-creation) prescribe other stakeholder involvement.

Disciplinary fields of 'Industrial Management' $(\mathrm{n}=$ 26), 'Human Computer Interaction' (HCI) $(\mathrm{n}=21)$, 'Knowledge Management' $(\mathrm{n}=17)$ and 'Engineering Research' $(\mathrm{n}=18)$ most frequently have drawn on LL conceptual /methodological papers. On inspection of citation data, interest from 'Industrial Management' and 'Engineering Research' largely aligns to dissemination of LL work at ICE/ITMC. Here, most activity for 'Engineering Research' appeared at these venues in $2013(\mathrm{n}=7)$ and $2015(\mathrm{n}=6)$. 'Industrial Management' related Research largely appeared at ICE/ITMC in the years $2010(\mathrm{n}=7), 2013(\mathrm{n}=7)$, and $2015(\mathrm{n}=6)$. HCI related papers have been most disseminated through 'Conference on Human Factors in Computing Systems' and 'Lecture Notes in Computer Science', appearing most often in 2013 ( $\mathrm{n}=$ 5) and 2015 (n $6=2015$ ). Figures suggest that there remains steady interest in LL research approaches amongst researchers within these domains.

A timeline breakdown of term bursts1 (bursts are indicative of increased interest within knowledge domains based on sudden increases in term or citation frequency) restricted to disciplinary field and/or 'research topic' are shown in figure 6. (displayed at the end the paper). It illustrates how LL work first gained sudden interest in 'Industrial Management' (a field of Business Administration concerning the structure and organization of industrial companies) and later garnered interest in the fields of 'Knowledge Management' and HCI. The most significant topic burst relates to 'Smart City', which began to emerge from 2011, with research on IoT technology, 'Health Care' and 'Virtual Reality' shortly after. More recently, it appears that 'Older/Aging adult' research,

1 Burst detection in Citespace is based on Kleinberg's algorithm [13] 
'Education' and 'Energy Efficiency' are topics that have begun to emerge as benefitting from LL approaches. However, no significant term bursts have appeared in recent years suggesting LL has not attracted major new interest by a field or 'research topic'. Though the visualization shows some initial recent interest in the disciplinary area of Ecology, more cross-disciplinary and international collaborations could help to spur additional interest in the benefits of LL approaches. As recently concluded by Maccani et al. [15], LL work has tended to be practice orientated, with the need to better align and formalize research within LLs according to disciplinary areas.

\subsection{How has the intellectual structure of $L L$ influenced research matured?}

Finally, to understand the scholarly community influenced by LL work, including the degree to which a clear Living Lab field(s) has developed, we used Citespace for Co-Citation Analysis by Author. Citespace allowed us to identify clusters of research by mapping network clusters of co-cited references by authors (Nodes and clusters are identified according to authors with similar referencing) [26]. We performed this on 'Dataset C' and their associated 15768 references. We set our criteria selection at the top 100 cited items and top $20 \%$ of cited items for each slice year. Figure 7 below represents the higher-level structure of the centre of the network, showing interconnected core clusters of research. (Small and unconnected ancillary surrounding clusters are not shown). Importantly, smaller clusters tend to be less representative, as they are based on the citing pattern of a fewer number of papers.

Altogether, Citespace identified 92 clusters of research, with just 14 clusters having five or more member nodes, and 11 clusters forming the core interconnected clusters (i.e. Figure 7). Member nodes refer to articles cited by papers in 'Dataset C'. The quality of a cluster is reflected in terms of its silhouette score, whereby a score closer to 1 indicates homogeneity. The modularity $\mathrm{Q}$ of the overall network structure is 0.8 , which is relatively high, suggesting the network is reasonably divided into loosely coupled clusters. Although the mean silhouette score of 0.23 means a measure of the homogeneity of these clusters on average is low, examining the top 10 clusters in Table 3. illustrates a high silhouette score for these clusters suggesting meaningful results. Examining the network structure suggests that most clusters do not have a connection to the evolution of knowledge domains drawing on LL work as they tend to be small, isolated and scattered across academic communities.
Table 3. Top 10 Cluster Groups

\begin{tabular}{|l|l|l|l|l|}
\hline Cluster & Size & $\begin{array}{l}\text { Sil } \\
\text { houette }\end{array}$ & $\begin{array}{l}\text { Mean } \\
\text { (Year) }\end{array}$ & Label (LLR) \\
\hline 0 & 43 & 0.771 & 2007 & living lab \\
\hline 1 & 32 & 0.785 & 2009 & $\begin{array}{l}\text { open } \\
\text { innovation }\end{array}$ \\
\hline 2 & 30 & 0.846 & 2010 & $\begin{array}{l}\text { radical service } \\
\text { innovation }\end{array}$ \\
\hline 3 & 23 & 0.839 & 2007 & living lab \\
\hline 4 & 22 & 0.919 & 2008 & $\begin{array}{l}\text { living lab } \\
\text { innovation }\end{array}$ \\
\hline 5 & 22 & 0.904 & 2008 & living lab \\
\hline 6 & 21 & 0.736 & 2008 & human sensor \\
\hline 7 & 20 & 0.92 & 2009 & user experience \\
\hline 8 & 18 & 0.965 & 2008 & $\begin{array}{l}\text { knowledge } \\
\text { valorization }\end{array}$ \\
\hline 9 & 14 & 0.998 & 2005 & $\begin{array}{l}\text { innovation } \\
\text { diffusion }\end{array}$ \\
\hline
\end{tabular}

Table 3. shows the top 10 clusters found according to node membership. Assigned cluster labels are based on keyword terms of papers citing member nodes using a log-likelihood ratio test method (LLR).2 The 'mean year' in the table refers to the average year of member node publication. For example, examining the largest cluster (Cluster 0), the 43 member nodes are generated from 65 (from Database C) papers co-citing node members within that cluster. Whereas the 'mean year' of member nodes is ' 2007 ', the average year of papers citing these nodes in the cluster is actually 2011. Importantly, analysis found that cluster 2 could be considered the youngest of the clusters in Table 3, whereby the average year of papers citing nodes in the cluster was 2014. Upon examination, it was found that such citing papers tend to focus on researching 'user typologies', 'user roles/patterns' and other factors affecting innovation in LLs.

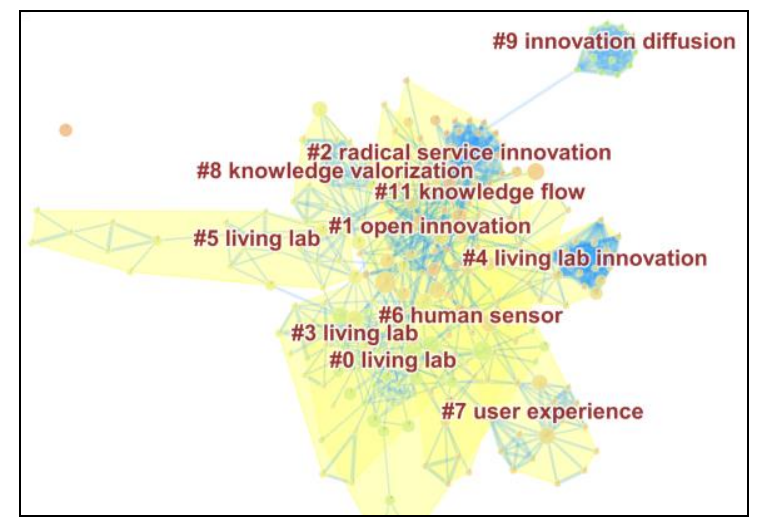

Figure 7. Core cluster network

2 Labels assigned to clusters are not necessarily representative of topic area. 
The 11 interconnected clusters (almost all of which are the largest clusters in Table 3) shown in Figure 7 form the core of the intellectual evolution of LL (influenced) research. We found no apparent cluster distinctly dominant in size with a high Silhouette score. This suggests that there lacks a cohesive approach to evolving the knowledge domain. Figure 8. illustrates the most significant authors to clusters, whereby Bergvall-Kareborn [3], Almirall [2] and Pallot [20] appear to have had the greatest impact in advancing the LL knowledge domain. Chesbrough [6], Von Hippel [11] and Mulder et al [17] have been drawn on in developing a theoretical/conceptual foundation for LL research in terms of 'Open Innovation', 'User Innovation' and 'Co-creation' respectively. Other relevant authors contributing research to LL not shown in fig. 83 include Ballon [e.g. 3], Leminen and Shuurman [e.g. 3].

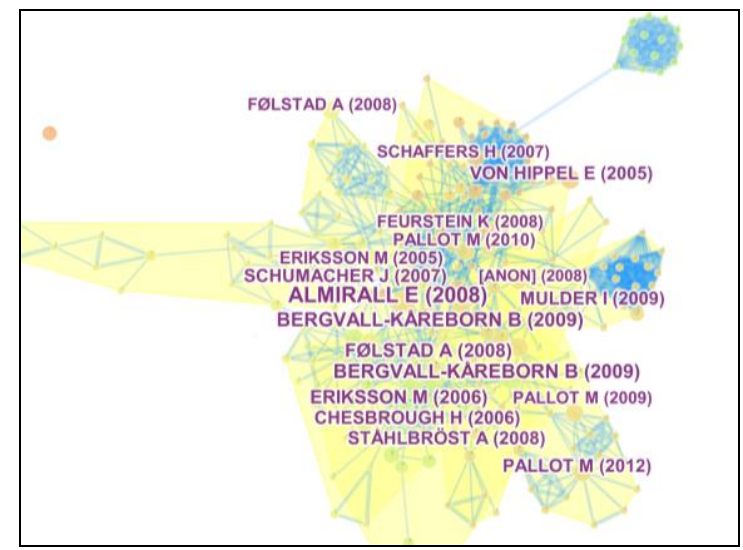

Figure 8. Top cited authors to Clusters

\section{Discussion and Conclusion}

Our analysis shows Living Lab as a concept and approach has been gaining increasing interest in the past 10 years in some cases exceeding (i.e. ADR) other approaches to innovation. Its relevance is likely to continue and perhaps grow based on its growing interest in the domain of 'Smart cities', ICT platform development, 'Internet of Things', 'Older adults' and 'Assisted Living'. Recent interest has also been shown in educational aspects to LL, design of sustainable products and services, mobile application development and energy efficiency research. Furthermore, LL would appear a fruitful approach to future technology development challenges in areas as, 'Autonomous Vehicle', 'Drone' and 'Augmented Reality' research, which require real-world experimentation and

3 Due to a citation threshold display of 10 being applied to Figure 8 . stakeholder involvement for deployment and scaling. As was shown from our findings, existing research output has been largely confined to Europe where significant EU funding efforts (including EnoLL) has spurred LL activity and thus related research dissemination (An example of a successful EuropeNon-Europe collaboration is the Beer Living Lab[22]). Given the nature of emerging themes suited to LL research listed above, there lies an opportunity to increase international LL research efforts in this regard.

However, our analysis clearly raises challenges for researchers in better establishing LL in the mainstream academic literature. These include: (1) the need to concentrate dissemination of LL work in suitable high impact publication venues receptive to LL work. (2) the need for European researchers (with expertise in this area) to better engage the international research community through cross-country collaborations. (3) and the necessity to concentrate efforts at evolving an intellectual core through consolidating efforts in developing a research agenda, both theoretical and empirical. With regards to each of these points (as previously reported in Maccani et al. (2017)), ADR holds promise in lending LL researchers a sound methodology to bolster research efforts and avenues of research dissemination, ultimately enhancing artifact design and scholarly impact. Whilst LL efforts tend to be more immediate and practice oriented, there lies an opportunity for LL researchers to pay closer attention to applying existing IS theory early in the design process, whilst formalizing learnings into theory validation/generation at the end. Furthermore, by relating LL to a DSR methodology, IS researchers have the opportunity of extending DSR to user driven open innovation environments.

Our analysis of 'Dataset C' showed the youngest of the significant clusters identified relates to research on understanding, 'user roles', 'actor roles' 'user typologies' etc. in the context of innovation through LL [e.g. 25], and it should be noted (based on our domain knowledge) that such interest is amongst the most significant of future calls for research in advancing LL literature. In this regard, ADR research could benefit from such existing insights when designing future studies in the context of open innovation environments, and in so doing could contribute to validating and advancing existing work.

Finally, future collaborations between ADR and LL researchers could enhance both; research agendas, research opportunities and research impact. Our study makes one such small step in assisting researchers (both ADR and LL) in their efforts to become involved in LL-ADR, and how best to contribute to such efforts. 


\section{Limitations}

'Database C' (i.e. $\mathrm{N}=496$ ) consists of papers citing 1 of the top 60 conceptual/methodological LL papers we identified. As these 60 top papers were collated based on a 'title' search for LL, this method may have served to exclude relevant papers for our analysis. An alternative method is to gather bibliometric data based on 'title, keyword, abstract' search for LL, though this method has implications of excluding relevant papers, as well as including irrelevant papers. Future work could compare and/or collate results from both search strategies in ensuring robust findings.

This work has been supported through funding from Science Foundation Ireland and Intel Corp.

\section{References}

[1] Almirall, E. and Wareham, J. Living Labs: Arbiters of mid- and ground-level innovation. Technology Analysis \& Strategic Management 23, July (2011), 87-102.

[2] Ballon, P. and Schuurman, D. Living labs: concepts, tools and cases. Info 17, 4 (2015), 1.

[3] Bergvall-Kåreborn, B., Ihlström Eriksson, Carina Ståhlbröst, A., and Svensson, J. A Milieu for Innovation Defining Living Labs. 2nd ISPIM Innovation Symposium, New York, November 2015 (2009), 6-9.

[4] Business, S. Defining Social Innovation. 2017. https://www.gsb.stanford.edu/faculty-research/centersinitiatives/csi/defining-social-innovation.

[5] Chen, C. CiteSpace II: Detecting and visualizing emerging trends and transient patterns in scientific literature. Journal of the American Society for Information Science and Technology 57, 3 (2006), 359-377.

[6] Chesbrough, H. Open Innovation: The New Imperative for Creating and Profitting from Technology. Harvard Business School Press Books, (2003).

[7] Coenen, T., Donche, V., and Ballon, P. LL-ADR: Action design research in living labs. Proceedings of the Annual Hawaii International Conference on System Sciences, (2015)

[8] EU. Future Internet Research and Experimentation. 2017. https://www.ict-fire.eu/.

[9] EU. Organicity. 2017. http://organicity.eu/.

[10] Haj-bolouri, A., Bernhardsson, L., and Rossi, M. Introducing PADRE : Participatory Action Design. Pre-ICIS Workshop, (2015).

[11] Von Hippel, E. Democratizing innovation: The evolving phenomenon of user innovation. Journal fur
Betriebswirtschaft 55, 1 (2005), 63-78.

[12] Keyson, D., Guerra-Santin, O., and Lockton, D. Living Labs: Design and Assessment of Sustainable Living. Springer, 2017.

[13] Kleinberg, J. Bursty and Hierarchical Structure in Streams. Data Mining and Knowledge Discovery, (2003), 373-397.

[14] Krogstie, J. Bridging research and innovation by applying living labs for design science research. Lecture Notes in Business Information Processing, (2012), 161-176.

[15] Maccani, G., Mcloughlin, S., Prendergast, D., and Donnellan, B. Positioning Living Labs within Action Design Research: Preliminary Findings from a Systematic Literature Review. 12th International Conference on Design Science Research in Information Systems and Technology (DESRIST), (2017).

[16] De Moor, K., Ketyko, I., Joseph, W., et al. Proposed framework for evaluating quality of experience in a mobile, testbed-oriented living lab setting. Mobile Networks and Applications 15, 3 (2010), 378-391.

[17] Mulder, I. and Stappers, P.J. Co-creating in practice: Results and challenges. 2009 IEEE International Technology Management Conference, ICE 2009, (2009).

[18] Niitamo, V.P., Kulkki, S., Eriksson, M., and Hribernik, K.A. State-of-the-art and good practice in the field of living labs. 2006 IEEE International Technology Management Conference, ICE 2006, (2006).

[19] Okoli, C. A guide to conducting a standalone systematic literature review. Communications of the Association for Information Systems 37, 1 (2015), 879-910.

[20] Pallot, M., Trousse, B., Senach, B., and Scapin, D. Living Lab Research Landscape : From User Centred Design and User Experience towards User Cocreation. Technology Innovation Management Review 1, (2010), 19-25.

[21] Price, D.D.S. A general theory of bibliometric and other cumulative advantage processes. Journal of the American Society for Information Science 27, 5 (1976), 292-306.

[22] Rukanova, Boriana; Baida, Ziv; Liu, Jianwei; Van Stijn, E. Beer living lab-intelligent data sharing. In Accelerating Global Supply Chains with IT-Innovation. Springer, Berlin, 2011, 37-54.

[23] Schuurman, D., De Moor, K., De Marez, L., and Evens, T. Investigating user typologies \& their relevance within a living lab-research approach for ICT-innovation.Proceedings of the Annual Hawaii International Conference on System Sciences, (2010).

[24] Thapa, D., Harnesk, D., Ståhlbröst, A., \& Padyab, A.M. Make way for the new wave: Living Labs as a DSR Approach. Information Systems Research Seminar in 
Scandinavia: IRIS 37: Designing Human Technologies, (2014).

[25] Thornley, C. V, McLoughlin, S.J., Johnson, A.C., and Smeaton, A.F. A bibliometric study of Video Retrieval Evaluation Benchmarking (TRECVid): A methodological analysis. Journal of Information Science 37, 6 (2011), 577-
593.

[26] White, H.D. and Griffith, B.C. Author cocitation: A literature measure of intellectual structure. Journal of the American Society for Information Science 32, 3 (1981), 163 171.

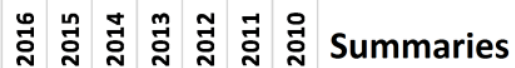

$\begin{array}{lllllllll}\text { service(s) } & 20 & 22 & 37 & 31 & 20 & 23 & 13 \text { Service design, development and innovation, experimentation/evaluation as a service }\end{array}$

\begin{tabular}{l|llllllll} 
Case (study) & 23 & 32 & 21 & 24 & 23 & 10 & 13 & Case study of living labs or innovation approaches, technology development case studies
\end{tabular}

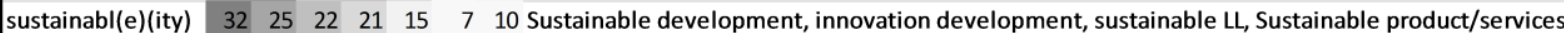

$\begin{array}{llllllllll}\text { Technolog(y)(ies) } & 17 & 16 & 17 & 18 & 12 & 13 & 15 & \text { Technology Innovation, technology commercialisation, technology design }\end{array}$

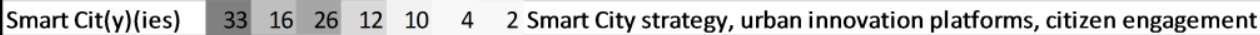

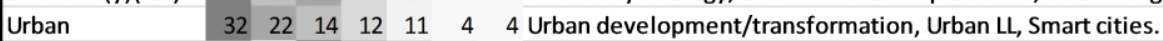

$\begin{array}{llllllll}\text { Open Innovation } & 17 & 5 & 20 & 11 & 10 & 9 & 4\end{array}$ Living Labs as an approach to Open innovation or Open Innovation processes in Living labs

$\begin{array}{lllllllll}\text { Learning } & 10 & 16 & 20 & 14 & 5 & 6 & 5 \text { Learnings from LL/innovation, methods of learning in LL/innovation, digitised/e-learning design }\end{array}$

$\begin{array}{lllllllll}\text { Education(al) } & 14 & 17 & 9 & 20 & 5 & 3 & 4 & \text { Education in Innovation, University technology transfer, LL as tool for education }\end{array}$

\begin{tabular}{llllll|l|l} 
Knowledge & 10 & 4 & 11 & 21 & 5 & 12 & 6 Knowledge exchange, creation, support
\end{tabular}

$\begin{array}{lllllllll}\text { Framework } & 11 & 12 & 8 & 11 & 9 & 8 & 8 & \text { Proposed theoretical/methodological/evaluation Framework }\end{array}$

$\begin{array}{lllllllll}\text { Mobile } & 6 & 14 & 8 & 10 & 4 & 10 & 11 & \text { Mobile participation in LL, Mobile App development }\end{array}$

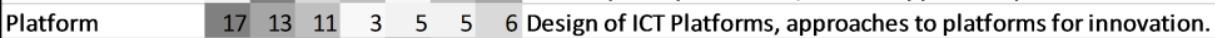

$\begin{array}{llllllll}\text { Business } & 7 & 6 & 10 & 7 & 12 & 9 & 8 \text { Business models for living labs, business model development via experiments }\end{array}$

$\begin{array}{lllllllll}\text { Aging/elderly } & 5 & 16 & 8 & 4 & 6 & 3 & 2 & \text { Elderly care, QoL, Virtual/gaming training and communication }\end{array}$

$\begin{array}{llllllllll}\text { Rural } & 2 & 3 & 2 & 5 & 3 & 6 & 21 & \text { Rural enterprise Innovation, LL fostering rural development }\end{array}$

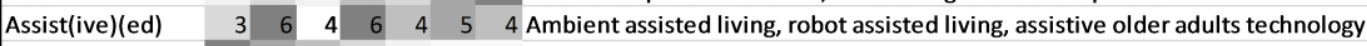

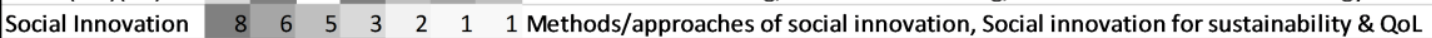

$\begin{array}{lllllllll}\text { Internet of Things } & 3 & 4 & 2 & 4 & 2 & 3 & 3 & \text { Internet of things technology development }\end{array}$

\begin{tabular}{lllllllll} 
Co Design & 4 & 4 & 4 & 2 & 0 & 1 & 1 Co-design approach \\
\hline
\end{tabular}

\section{Figure 4. Top 20 terms obtained from Dataset B}

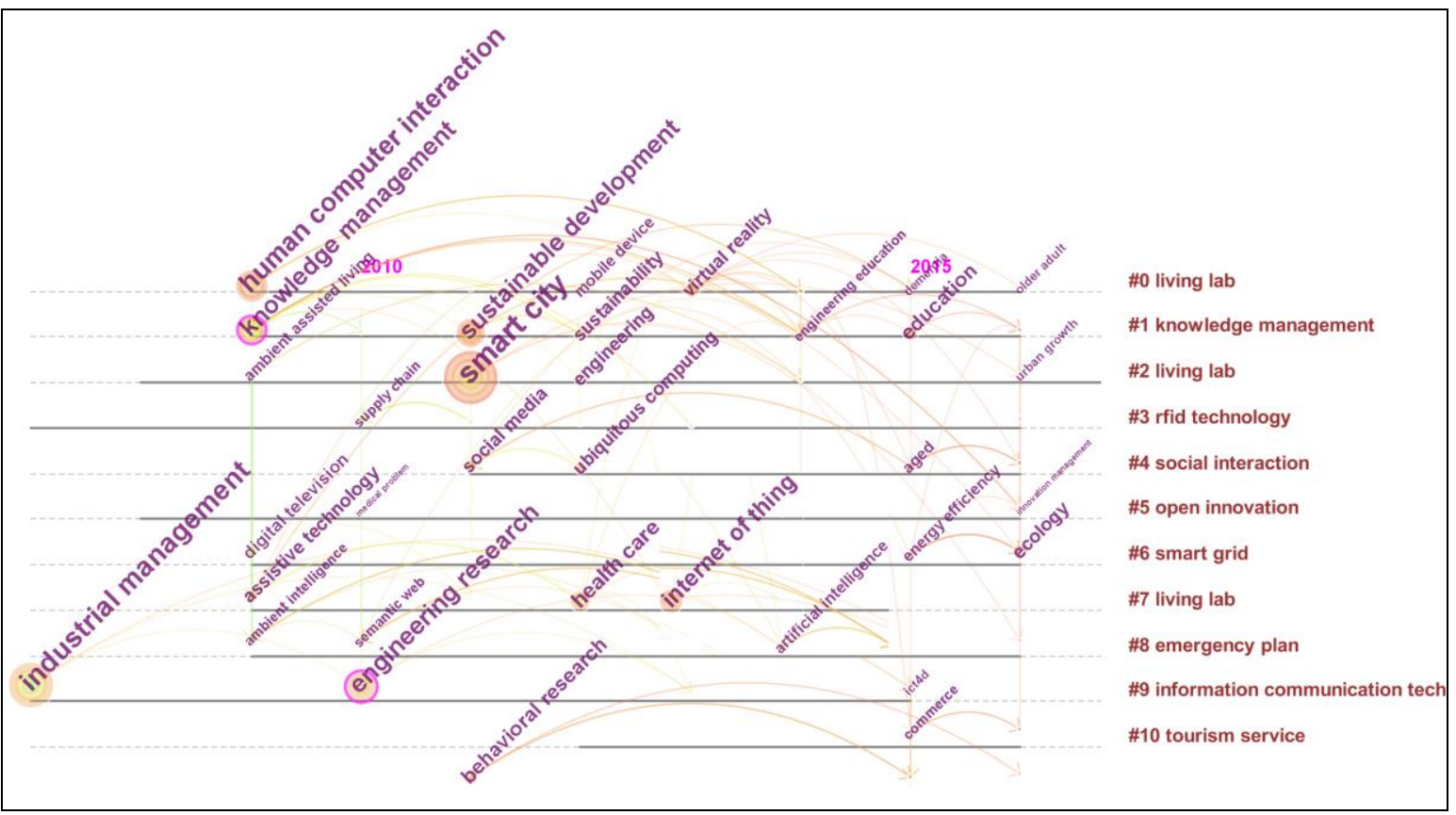

Figure 6. Discipline and Research topic term evolution using Citespace 